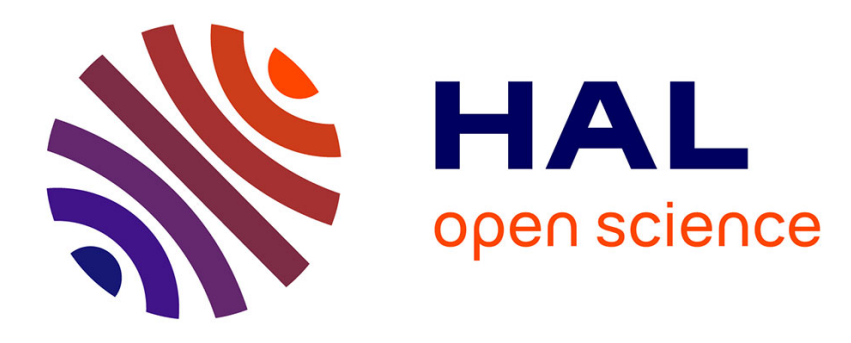

\title{
The many-defect problem : gauge-like variables for ordered media containing defects
}

B. Julia, G. Toulouse

\section{To cite this version:}

B. Julia, G. Toulouse. The many-defect problem : gauge-like variables for ordered media containing defects. Journal de Physique Lettres, 1979, 40 (16), pp.395-398. 10.1051/jphyslet:019790040016039500 . jpa-00231652

\section{HAL Id: jpa-00231652 https://hal.science/jpa-00231652}

Submitted on 1 Jan 1979

HAL is a multi-disciplinary open access archive for the deposit and dissemination of scientific research documents, whether they are published or not. The documents may come from teaching and research institutions in France or abroad, or from public or private research centers.
L'archive ouverte pluridisciplinaire HAL, est destinée au dépôt et à la diffusion de documents scientifiques de niveau recherche, publiés ou non, émanant des établissements d'enseignement et de recherche français ou étrangers, des laboratoires publics ou privés. 


\title{
LE JOURNAL DE PHYSIQUE-LETTRES
}

Classification

Physics Abstracts

$12.90-64.90$

\section{The many-defect problem : gauge-like variables for ordered media containing defects}

\author{
B. Julia
}

Laboratoire de Physique Théorique de l'Ecole Normale Supérieure $\left(^{*}\right)$

and G. Toulouse

Laboratoire de Physique de l'Ecole Normale Supérieure.

(Reçu le 12 avril 1979, accepté le 27 juin 1979)

\begin{abstract}
Résumé. - Il serait souhaitable de disposer d'une théorie générale pour les milieux ordonnés contenant une distribution continue de défauts. La théorie de I. Dzyaloshinskii et G. Volovik est discutée. On propose un autre modèle fondé sur des considérations topologiques.
\end{abstract}

\begin{abstract}
A general theory for ordered media containing a continuous distribution of defects is desirable. The hydrodynamic theory of I. Dzyaloshinskii and G. Volovik is discussed. Another proposal stressing topological considerations is presented.
\end{abstract}

The classification of defects in ordered media (the one-defect problem) has much advanced recently thanks to the use of homotopy theory. This also improved the understanding of combination laws of defects (the two-defect problem). Recently, I. Dzyaloshinskii and G. Volovik [1] have put forward an approach to the many-defect problem based on gauge theory concepts, which contains both a reappraisal of ancient ad hoc theories for the hydrodynamics of superfluid helium 4 containing vortices [2] or for the elasticity of crystals containing dislocations [3], and suggestions for a generalization to other ordered media, involving the introduction of gauge fields for the full original (global) symmetry group of the symmetric phase, called here-after the broken symmetry group. However, this generalization scheme leads to some difficulties concerning the counting of supplementary long-lived degrees of freedom related to a continuous distribution of defects, furthermore it does not tackle quite properly the description of defects which are of a different type from that of lines in three-dimensional space (points or non singular configurations, etc.).

(*) Laboratoire propre du C.N.R.S. associé à l'Ecole Normale Supérieure et à l'Université de Paris-Sud. Postal address : 24, rue Lhomond, 75231 Paris Cedex 05, France.
We thus consider here a different generalization scheme where, instead of the gauge fields of the broken symmetry group, one introduces gauge-like variables related to the homotopy groups of the manifold of internal states (characteristic of the broken symmetry phase). In order to show the physics of this programme, it is useful to reconsider first the basic example of superfluid helium 4, from which both generalization schemes can be developed.

1. The case of superfluid helium 4. - In this case, the broken symmetry group is $\mathrm{G}=\mathrm{U}(1)$, the unbroken subgroup is $\mathrm{H}=1$, the manifold of internal states is $\mathrm{V}=\mathrm{G} / \mathrm{H}=\mathrm{S}_{1}$, and the only nontrivial homotopy group is $\Pi_{1}\left(S_{1}\right)=\mathrm{Z}$, describing vortex lines.

In the absence of vortices, the phase of the order parameter is a one-valued function ; the superfluid velocity can be defined as its gradient, whose circulation around a closed contour is zero. In the presence of a vortex, the phase of the order parameter is a multivalued function and the topological index of the vortex is obtained as the circulation of the superfluid velocity (which is no longer a gradient everywhere) along a surrounding loop. In the presence of many vortices, the hydrodynamic theory claims that one should consider the three cuinpo- 
nents of the superfluid velocity as independent variables, that means adding 2 more variables, from which one can extract a density and a current of defects, obeying automatically some suitable conservation laws. But before showing this, for later purposes of generalization, let us first introduce the language of differential forms [4].

A 0 -form is a real scalar function; a 1-form is a vector ; a 2-form is an antisymmetric tensor, ... The exterior derivative of a $p$-form is a $(p+1)$-form whose components are antisymmetrized gradients (curls) if

$$
\begin{gathered}
A=A_{i j} \ldots \mathrm{d} x^{i} \wedge \mathrm{d} x^{j} \wedge \ldots, \\
\mathrm{d} A=F=\partial_{m} A_{i j} \ldots \mathrm{d} x^{m} \wedge \mathrm{d} x^{i} \wedge \mathrm{d} x^{j} \ldots
\end{gathered}
$$

The second exterior derivative of a form is zero : $\mathrm{d}^{2} \omega=0$. A form $\omega$ is closed if $\mathrm{d} \omega=0$. A form $\omega$ is exact if $\omega=\mathrm{d} \Omega$, where $\Omega$ is another form. The number $N$ of components of a $p$-form in $d$-dimensional space is $C_{d}^{p}$. Since this will be of repeated use in the following, table I gives this number of components for $d=1,2,3,4$.

Table I. - Number of components of a p-form in $d$ dimensional space.

\begin{tabular}{|c|c|c|c|c|}
\hline$p$ & 1 & 2 & 3 & 4 \\
\hline 0 & 1 & 1 & 1 & 1 \\
\hline 1 & 1 & 2 & 3 & 4 \\
\hline 2 & & 1 & 3 & 6 \\
\hline 3 & & & 1 & 4 \\
\hline 4 & & & & 1 \\
\hline
\end{tabular}

We shall have mainly in mind 3-dimensional materials, but lower dimensionality models are readily considered and often instructive. It is generally convenient to consider together time and space components $(D=3+1)$, although Lorentz invariance is not manifest in condensed matter physics. It is important to note that exterior differentiation does not involve the (Lorentz) metric.

Coming back to superfluid helium 4 , one considers first the medium in the absence of vortices. The variable is a 0 -form : the phase $\varphi$. The superfluid velocity is a 1-form, and as it is an exact form, its exterior derivative vanishes (table II). For the medium with vortices, the hydrodynamic theory shifts down the whole pattern by one rung of the ladder.

\begin{tabular}{|c|c|c|c|c|}
\hline \multirow[b]{2}{*}{$p$} & \multicolumn{2}{|c|}{$\begin{array}{c}\text { Number } \\
\text { of components }\end{array}$} & \multirow[b]{2}{*}{ No vortex } & \multirow[b]{2}{*}{ Many vortices } \\
\hline & $\begin{array}{l}\text { Space } \\
\text { only }\end{array}$ & $\begin{array}{c}\text { Space }+ \\
\text { time }\end{array}$ & & \\
\hline 0 & 1 & 1 & Variable $\varphi$ & \\
\hline 1 & 3 & 4 & $\begin{array}{l}\text { Superfluid velocity } \\
v, \mathrm{~d} x=\mathrm{d} \varphi\end{array}$ & Variables $A_{\mu}$ \\
\hline 2 & 3 & 6 & $d^{2} \varphi=0$ & $\begin{array}{c}\text { density and current } \\
F_{\mu_{\nu}}\end{array}$ \\
\hline 3 & 1 & 4 & & conservation laws \\
\hline
\end{tabular}

Table II. - Hydrodynamic variables for $\mathrm{He}_{4}$.

The idea is to replace a closed form, defined outside of the many defects (that is on a very complicated manifold), by a 1-form, defined everywhere but otherwise arbitrary. Remember that in this case the hydrodynamic variables are the 3-space components $\mathbf{A}$. The information on the defect network is now contained in the exterior derivative of the 1-form $A=A_{\mu} \mathrm{d} x^{\mu}$, which is a 6-component twoform : $F=-1 / 2 F_{\mu \nu} \mathrm{d} x^{\mu} \wedge \mathrm{d} x^{\nu}$, with 3 pure space components giving the density of vortices $\mathbf{h}$, and 3 mixed components corresponding to the current of vortices : e. This interpretation includes the vortex free situation : if $F$ vanishes, the 1 -form $A$ must be exact according to Poincaré's theorem (the medium is assumed to be simply connected), and can be defined everywhere. In the general case, the exterior derivative of the exact 2 -form $F$ is a vanishing 3-form ; this yields 4 identities, which may be written as

$$
\left\{\begin{array}{l}
\operatorname{div} \mathbf{h} \equiv 0 \\
\operatorname{rot} \mathbf{e}+\frac{\partial \mathbf{h}}{\partial t} \equiv 0 .
\end{array}\right.
$$

The first equation involves $\mathbf{A}$ only and says that linevortices do not terminate; the second equation is a conservation law for lines and holds for any choice of $A_{0}$. The interpretation of $\mathbf{e}$ as a current suggests a particular choice for $A_{0}$ [1] such as to obtain the constitutive equation :

$$
\mathbf{e}+\mathbf{v} \wedge \mathbf{h}=\mathbf{0}
$$

for vortices moving with the normal fluid velocity $\mathbf{v}$, by analogy with Euler's equation. The choice of $A_{0}$ is also restricted by requiring the current to vanish in the absence of vortices $(A=\nabla \varphi)$ or in the presence of static ones.

The electromagnetic notation (h, e) has been chosen to stress the analogy of eq. (1) with the homogeneous Maxwell equations; geometrically (1) is nothing but a Bianchi identity and implies the existence of 1-forms $A$ (up to gauge transformations) whose exterior derivative is a given $F$. For a reasonable definition of the current $e$, one expects the constitutive equation to admit a gradient expansion and 
from (1) one can infer that the new (topological, additive) variable $\mathbf{h}$ must be hydrodynamical and must lead to additional slow modes.

In summary, the existence of a non-trivial first homology group : $\mathrm{Z}$ (related to the classification of differential forms [4]) implies, in the many-defect limit, the existence of a slowly varying quasi-continuous magnetic flux $\mathbf{h}$. The introduction of the gauge field 1-form $A$ (with values in the Lie algebra of U(1)) allows one to describe the topological stability of the line defects in superfluid helium 4 by kinematic equations which are identically satisfied. In a second stage, one must derive, from an appropriate Hamiltonian, the dynamic equations and finally introduce dissipation terms [1].

Let us mention that, in the case of crystalline lattices with translation dislocations, the whole discussion can be repeated, with the minor modification that now $\Pi_{1}=\mathrm{H}_{1}=\mathrm{Z}^{3}$; the topological index is a Burgers vector with 3 components, the gauge algebra is that of $\mathrm{U}(1) \times \mathrm{U}(1) \times \mathrm{U}(1)$, wo that the total number of components of the various $p$-forms has to be multiplied by 3 : the number of generators of the gauge group.

2. Discussion. - The main step in the previous section consists in the replacement of a gradient field $\nabla \varphi$ by a general vector field $\mathbf{A}$, in order to describe the medium with vortices.

This is mathematically reminiscent of the transition from a liquid to a solid where, in addition to one longitudinal propagating sound mode, one gets two extra transverse modes. As pointed out by Virasoro (private communication), there is also some analogy with the Higgs phenomenon in superconductors, where the scalar phase of the matter field becomes part of a vector field, through coupling to the electromagnetic field.

This replacement procedure has two attractive features, and one may say that it has both kinematic appeal and dynamic appeal.

It has kinematic appeal because the new variables automatically yield the suitable conservation laws for stable line defects. It has dynamic appeal because the replacement ensures that the theory without vortices is embedded inside the theory with vortices and, as a consequence, it provides some guidance for building the larger theory.

In a general theory of ordered media containing defects, one would like to keep together these two attractive features. But is it possible ?

The Dzyaloshinskii-Volovik scheme, as we see it, follows the dynamic appeal. It introduces as new variables the gauge fields $A_{\mu}^{a}(a=1, \ldots, g$ is an isotopic index) associated with the Lie algebra of $G$, the broken symmetry group $G$. Generally, $G$ will be a non-abelian group, with $g$ generators, and the theory will possess non-linear features, as considered by Yang and Mills.
Another proposal suggests itself naturally, following the kinematic appeal. Then one would introduce as new variables the gauge-like fields (forms of various order) related to the homotopy groups $\Pi_{r}(\mathrm{~V}=\mathrm{G} / \mathrm{H})$ which describe the topologically stable defects, via homology groups (topological charges).

First, let us discuss the Dzyaloshinskii-Volovik (D.V.) scheme, and remark that it loses contact, in general, with the topology. This is so because the variables introduced there are only determined by $G$, the broken symmetry group, whereas the topological classification of defects is determined both by $G$ and $H$, the unbroken subgroup. Therefore, for instance, the ferromagnet, the noncolinear antiferromagnet or the nematic, when containing defects, would be described by the same number of variables, because they have same $\mathrm{G}=\mathrm{SO}(3)$, despite the fact that their topologically stable defects are of widely distinct nature : in each case, the D.V. scheme introduces 6 new transverse variables (because $\mathrm{SO}(3)$ has 3 generators). In the case of nematics, the homotopy groups indicate that there may exist stable line and point defects : but the D.V. scheme would introduce the same number of variables to describe a nematic with line defects only, a nematic with point defects only, or a nematic containing both line and point defects.

Furthermore, the variables introduced (gauge fields $A_{\mu}^{a}$ ) are fit to describe topological line defects. This has been shown by our analysis, in the previous section, for the abelian case of helium 4 . For a general non-abelian gauge group, there is also a Bianchi identity which generalizes the set of eqs. (1). For non-topological line defects, or for point defects, there seems to be no a priori justification why the associated variables should automatically obey such conservation laws.

This discussion is of some importance because the main step, perhaps, in a hydrodynamic approach, consists in making a proper counting of the number of long-lived degrees of freedom. Quickly relaxing (or frozen) degrees of freedom are not relevant variables for hydrodynamics [5]. Topological stability, as measured by the homotopic analysis, seems to be a reasonable criterion for the long-lived nature of defects, although it may not be the only one (see for instance the discussion on vacancies in Ref. [4]).

3. Gauge-like variables for topologically stable defects. - In the previous section, we mentioned that a different scheme, for a generalized hydrodynamics of ordered media containing defects, was conceivable. In this scheme, the variables are chosen so as to yield automatically the conservation laws for the defects which happen to be topologically stable. In the case of superfluid helium 4 with vortices, as discussed in section 1 , both schemes will coincide 
because $\mathbb{R}$ is the Lie algebra of $\mathrm{G} / \mathrm{H}$ as well as the macroscopic average of $\mathrm{Z}$, the first homotopy group. But, in general, they will differ. For instance, different variables will be introduced to describe a medium with point defects, because the conservation laws for point and line defects are different.

Generally, for topological defects of dimensionality $(d-r-1)$ associated with a non trivial $\Pi_{r}$, one should introduce $(r+1)$-forms $F$ which are closed (conservation laws $\mathrm{d} F=0$ ), defined everywhere (in a macroscopic limit) and which derive from gauge fields $A$ ( $r$-forms).

For instance, for point defects in ferromagnets (or nematics), the variables introduced will belong to a 2 -form $A$, whose expression outside of the defects is

$$
A_{\mu \nu}=\frac{1}{8 \pi} \varepsilon^{a b c} \psi_{a} \partial_{\mu} \psi_{b} \partial_{v} \psi_{c}
$$

where $\psi_{a}\left(a=1,2,3 ; \sum_{a} \psi_{a}^{2}=1\right)$ is the order parameter. Actually, the physics of the point defects will be related to the non-pure-gauge, or gaugeinvariant, part of the 2 -form $A$ (i.e. $F$ ). The variables so introduced are derived from the homotopy group $\Pi_{2}\left(\mathrm{~S}_{2}\right)=\mathrm{Z}$, (or $\Pi_{2}\left(\mathrm{P}_{2}\right)=\mathrm{Z}$, for nematics), in two respects. Because $\Pi_{2}$ is a second homotopy group, the variables $A_{\mu \nu}$ belong to a 2 -form. Because $\mathrm{Z}$ is an abelian group, these variables do not carry an isotopic index nor any of the non-linear features of YangMills theories.

If the homotopy group considered is isomorphic to $\mathrm{Z}$ or to powers of $\mathrm{Z}$, this procedure is relatively straightforward. However, if it is isomorphic to $Z_{2}$ for instance, a difficulty arises because this group is discrete. Mathematically, this touches the relations between homology and differential forms, and how much of the homotopy content they can describe. Physically, one may comment that defects described by a $\mathrm{Z}_{2}$ homotopy group are fermion-like, and that no macroscopic variable can be associated with them (whereas defects described by a $\mathrm{Z}$ homotopy group are boson-like). But this statement leaves a feeling of unsatisfaction because such defects can be of physical importance and one would not be entirely happy with a treatment which would not include them. Besides, all the noncommutative features which have been studied in the two-defect problem (noncommutative $\Pi_{1}$, action of $\Pi_{1}$ on $\Pi_{2}$, Whitehead products [6]) have yet to find their proper expression in this formulation of the many-defect problem.

4. Perspectives. - The generalization of hydrodynamics to ordered media (without defects) has been much discussed during the last decade. It has been recognized that each continuous broken symmetry, related to the ordering in the medium, leads to a supplementary long-lived degree of freedom (hydrodynamic variable) [5]. However all the consequences of this theory, such as the existence of a vacancy diffusion mode in solids, have not yet been checked experimentally [7].

I. Dzyaloshinskii and G. Volovik [1] deserve credit for opening the study of the generalized hydrodynamics of ordered media containing defects. As discussed here, the theory requires further clarification and it appears already as a promising problem in condensed matter physics, with potential implications in mathematics and particle physics. Thus it is interesting to compare our general formalism with a model studied in particle physics [8], where the topological charge is the integral of a 3 -form at infinity $(d=4)$, the fact that the gauge like variable is longranged corresponding in our language to its hydrodynamic character.

Acknowledgments. - One of us (B.J.) would like to thank the Commissariat à l'Energie Atomique for its magnanimous hospitality and the other (G.T.) his colleagues I. Dzyaloshinskii, G. Volovik and V. Mineyev for friendly discussions.

\section{References}

[1] DzyaloshinskiI, I., Volovik, G., J. Physique 39 (1978) 693 ; Volovik, G., Dzyaloshinskil, I., Zh. ETF (1978) 1102 ; DzyaloshinskiI, I., Volovik, G., Poisson Brackets in Condensed Matter Physics, to appear in Phys. Rev.

[2] See, for instance, Khalatnikov, I. M., Introduction to the Theory of Superfluidity (Benjamin) 1965.

[3] See, for instance, Landau, L., LifCHITz, E., Théorie de l'Elasticité (Mir) 1967.

[4] Flanders, H., Differential Forms (Academic Press, N.Y.) 1963 ;

Choquet-Bruhat, Y. et al., Analysis, Manifolds and Physics (North-Holland Amsterdam) 1977.

[5] Martin, P. C., Parodi, O., Pershan, P. S., Phys. Rev. A 6 (1972) 2401

[6] Poénaru, V., Toulouse, G., J. Physique 38 (1977) 887 ; J. Math. Phys. 20 (1979) 13.

[7] Fleming III, P. D., Cohen, C., Phys. Rev. B 13 (1976) 500.

[8] LÜSCHER, M., Phys. Lett. 78B (1978) 465. 